\title{
A microscopic study of the corneal stromal lenticules extracted during femtosecond laser-assisted small incision lenticule extraction
}

\author{
YEWEI YIN ${ }^{1,2}$, TU HU ${ }^{1,2}$, AIQUN XIANG ${ }^{1}$, YANYAN FU ${ }^{1}$, YANG ZHAO $^{1}$, \\ XIAOYING WU ${ }^{3}$, XIAOYING WU ${ }^{1,2}$ and DAN WEN ${ }^{1,2}$ \\ ${ }^{1}$ Eye Center of Xiangya Hospital, Central South University; ${ }^{2}$ Hunan Key Laboratory of Ophthalmology; \\ ${ }^{3}$ Department of Pathology, College of Basic Medicine, Central South University, Changsha, Hunan 410008, P.R. China
}

Received October 22, 2019; Accepted November 20, 2020

DOI: $10.3892 /$ etm.2021.10113

\begin{abstract}
The aim of the present study was to investigate the histological and morphological characteristics of corneal stromal lenticules extracted during femtosecond laser-assisted small incision lenticule extraction (SMILE) surgery by light and electron microscopy. A total of 20 human corneal stromal lenticules extracted during SMILE surgery were sent for microscopic examination immediately after surgery. Of these, six were observed under a light microscope and 14 were observed under an electron microscope. The smoothness of the front and rear surface of the lenticules observed under an electron microscope was rated on a scale of 0 to 4 according to unified evaluation criteria and the scores were statistically compared. Under the light microscope, the edge of the cross section of the corneal stromal lenticules was deeply stained and certain burrs and broken collagen fibers were observed. The swollen corneal stromal fibers were distributed irregularly, with a few bubbles of different sizes. Under the electron microscope, the surface of the lenticules ablated using a femtosecond laser was not perfectly smooth and the front surface was smoother than the rear surface. The side edge of the lenticules ablated using a femtosecond laser was not as regular as the edge cut using microscissors. Necrosis and dissolution of collagen fibers were observed near the surface of the lenticules. In conclusion, the surface quality of corneal stromal lenticules ablated using a femtosecond laser was not optimal when observed under a microscope. Increased attention should be paid to the histology and morphology of the corneal surface following laser ablation.
\end{abstract}

Correspondence to: Professor Dan Wen, Eye Center of Xiangya Hospital, Central South University, 87 Xiangya Street, Changsha, Hunan 410008, P.R. China

E-mail:wendan@csu.edu.cn

Key words: small incision lenticule extraction, corneal stromal lenticule, histology, morphology, surface quality, microscopy

\section{Introduction}

The introduction of femtosecond laser-assisted small incision lenticule extraction (SMILE) surgery has been a great advance in corneal refractive surgery in recent years (1-3). In order to correct the refractive error, SMILE makes use of a femtosecond laser to create a lenticule in the corneal stroma under the corneal cap, and then the lenticule is removed by a small peripheral incision (4-6). As a type of ultra-short pulse laser based on chirped pulse amplification technology, the femtosecond laser is considered to have high instantaneous power and minimal thermal effects, which makes it safer and more accurate compared with the excimer laser and the micromechanical knife (7).

However, studies that have been performed to investigate the surface quality of the corneal stroma following femtosecond laser ablation during SMILE surgery are currently limited. It has been hypothesized that the surface quality of the corneal stroma after laser ablation, in terms of smoothness and regularity, may directly affect visual quality following corneal refractive surgery $(8,9)$. In general, good surface quality after surgery may alleviate aberration and help achieve a reasonable visual quality. Due to the limited sources of human corneal tissue, the majority of previous studies investigating the surface quality after corneal refractive surgery focused on animal eyes (10). As an alternative to this, the corneal stromal lenticule extracted during SMILE surgery offers the possibility to study surface quality directly after femtosecond laser ablation.

Therefore, the present study aimed to preliminarily investigate the surface quality of the corneal stromal lenticule by using optical instruments, to explore corresponding influencing factors and to provide a morphological basis for the future improvement of femtosecond laser-assisted corneal refractive surgery.

\section{Materials and methods}

Subjects and surgical procedure. A total of 20 patients with myopia who underwent SMILE surgery were enrolled in the present study, including 9 males and 11 females aged 
between 18 and 30 years. The present study was approved by the Ethics Committee of Xiangya Hospital (Changsha, China). The surgery was performed in October 2018 at the Laser Treatment Center of the Ophthalmology Department of Xiangya Hospital (Changsha, China). The spherical diopter (D) was between -6.00 and $-8.75 \mathrm{D}(-7.01 \pm 0.71 \mathrm{D})$ and the columnar $\mathrm{D}$ was between -0.25 and $-2.50 \mathrm{D}(-1.33 \pm 0.53 \mathrm{D})$. Prior to surgery, written informed consent was obtained from the patients with myopia in accordance with the institutional guidelines following full comprehension of the benefits and risks of the surgery as well as the use of their cornea tissues for experimental purpose.

The surgery was performed by an experienced surgeon using the VisuMax femtosecond laser system (Zeiss AG). The surgery methods were as follows: i) The patients' refractive degrees and ablating thickness were checked, the surgery area was conventionally disinfected and the eyelid opener was placed following surface anesthesia with Alcaine; ii) accurate suction of the cornea was performed with a negative pressure suction device, the corneal stromal lenticule was created and an incision was made using a femtosecond laser; and iii) the lenticule was separated and extracted using the operating microscope. The surgery parameters were as follows: Cutting diameter, 6.0-6.8 $\mathrm{mm}$; thickness of the corneal cap, $120 \mu \mathrm{m}$; point spacing, $4.5 \mu \mathrm{m}$; row spacing, $4.5 \mu \mathrm{m}$; laser energy, 130 nJ. Following surgery, bitobramycin and dexamethasone eye drops were applied.

After extraction, the front surfaces of the corneal stromal lenticules were stained with gentian violet solution. Subsequently, the lenticules were flattened and kept in glass vials with $4 \%$ polyformaldehyde phosphate buffer solution for light microscopy or $2.5 \%$ glutaraldehyde for electron microscopy. Afterwards, the lenticules were immediately sent to the laboratory for further microstructural observation.

Histological section and microscopy examination. A total of six corneal stromal lenticules were fixed in $4 \%$ polyformaldehyde phosphate buffer solution for $24 \mathrm{~h}$ at $4^{\circ} \mathrm{C}$. Conventional dehydration, paraffin-embedding and slicing were performed, with a slice thickness of $4.5 \mu \mathrm{m}$. After H\&E staining, dehydration, transparentization with xylene and sealing, the slices were observed under a light microscope.

Electron microscopic observation and surface evaluation. For electron microscopic examination, 14 corneal stromal lenticules were fixed in $2.5 \%$ glutaraldehyde at room temperature for $24 \mathrm{~h}$ and in $2 \%$ osmium acid for $2 \mathrm{~h}$ with washing steps in between. The specimens were dehydrated in a gradient series of aqueous ethanol solution (50, 70, 90 and 100\%) and then in pure acetone. For the scanning electron microscopy (SEM) examination, the specimens were then placed in amyl acetate. After dehydration, the lenticule specimens were dried using a critical point dryer (model, HCP-2; Hitachi, Ltd.) with liquid $\mathrm{CO}_{2}$. Subsequently, the specimens were mounted on aluminum stubs and sputtered with gold. Finally, the Hitachi HT7700 transmission electron microscope and the Hitachi S-3400nN SEM (Hitachi, Ltd.) were used to observe the slices.

To compare the quality of the front and rear surfaces of the lenticules, the electron microscopy images of the samples with a scale bar of $500 \mu \mathrm{m}$ were rated according to unified criteria
Table I. Evaluation criteria of the surface quality of corneal stromal lenticules observed under a scanning electron microscope.

\begin{tabular}{ll}
\hline Smoothness score & \multicolumn{1}{c}{ Microscopic observation } \\
\hline 1 & $\begin{array}{l}\text { Very rough: No obvious area without } \\
\text { burrs, curls or tissue bridges } \\
\text { Rough: More than half of the surface } \\
\text { has burrs, curls or tissue bridges } \\
\text { Smooth: Over half the surface has no } \\
\text { obvious burrs, curls or tissue bridges } \\
\text { Very smooth: No obvious burrs or curls }\end{array}$ \\
\hline
\end{tabular}

(Table I) (11). The surface quality of the lenticules was graded as follows: 1 , very smooth; 2 , smooth; 3 , rough; 4 , very rough. All electron microscopy images were anonymized and judged in a double-blinded manner by three technicians independently who were trained in electron microscopy image analysis. The average of the score determined by the three technicians was considered to be the final rating of each electron microscopy image.

Statistical analysis. Measurement data were expressed as the mean \pm standard deviation. The SPSS v23.0 statistical software package (IBM Corp.) and a paired t-test were used for the statistical analysis of the front and rear surface quality scores. $\mathrm{P}<0.05$ was considered to indicate a statistically significant difference.

\section{Results}

Histological appearance of the corneal stromal lenticules under the light microscope. Using a light microscope, the cross section of the lenticule was observed as illustrated in Fig. 1. The following was observed: i) The thickness of the lenticules was inconsistent, which may have been caused by the swelling of collagen fibers to varying degrees; ii) the edge of the lenticule, particularly the rear surface, was irregular with burrs and broken fibers; iii) the edge of the lenticule, particularly the anterior surface, was deeply stained, which indicated that the laser energy had damaged the corneal stroma; iv) the collagen fibers were distributed irregularly and stained unevenly, and certain nucleated corneal stromal cells were sprinkled throughout the collagen bundles; and v) bubbles of different sizes were visible throughout the collagen bundles without distribution differences and certain bubbles were connected with others.

Observation of the corneal stromal lenticules under the electron microscope. The microstructure, as observed under an electron microscope, is presented in Figs. 2 and 3. The following observations were made: i) Linear uplifts, short wrinkles and curls were present on the front and rear surfaces (Fig. 2A-D); ii) the front surface was relatively smooth (Fig. 2A and B), whereas the rear surface exhibited a certain number of tissue bridges and fractured collagen fibers (Fig. 2C and D); iii) the 
A

R

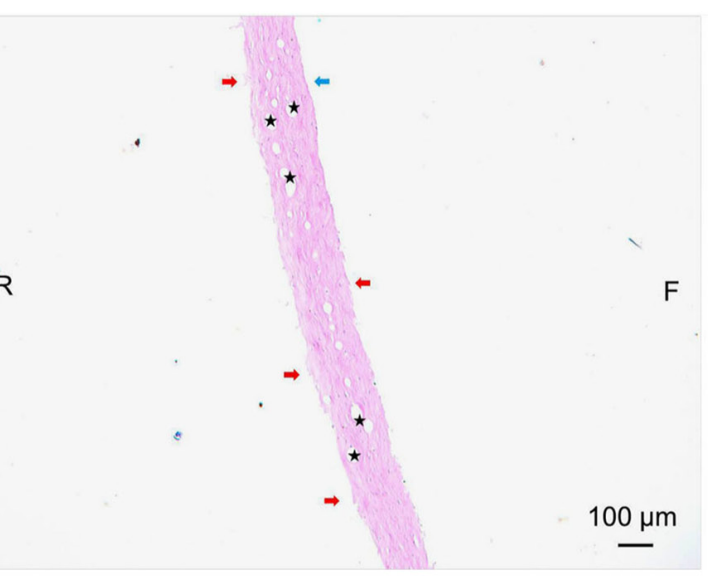

B

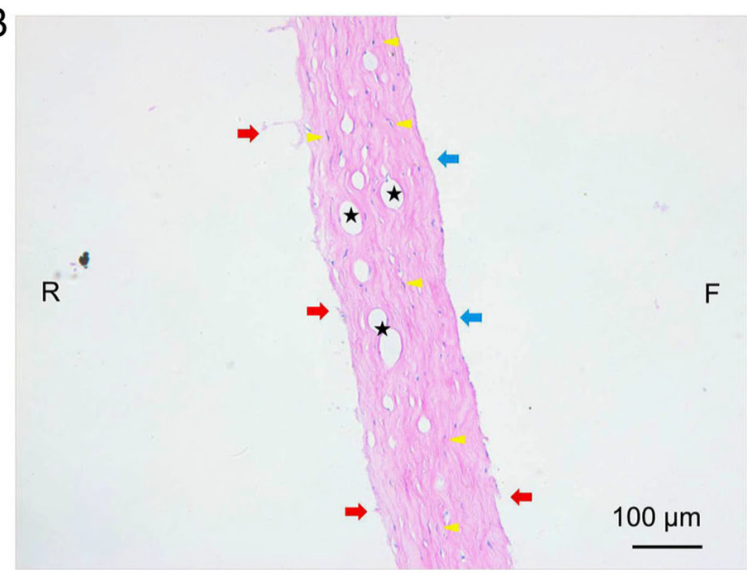

Figure 1. Under the light microscope, some burrs and broken fibers (red arrows) were observed at the edge of the cross section. Some of the edge tissues were deeply stained (blue arrows). The collagen fibers were stained unevenly, with some nucleated corneal stromal cells (yellow triangles) and bubbles of different sizes (black asterisks). (A) The inconsistent thickness of the lenticule. (B) The enlarged view of the area marked by the small black box in (A). F, front surface; $\mathrm{R}$, rear surface.

A

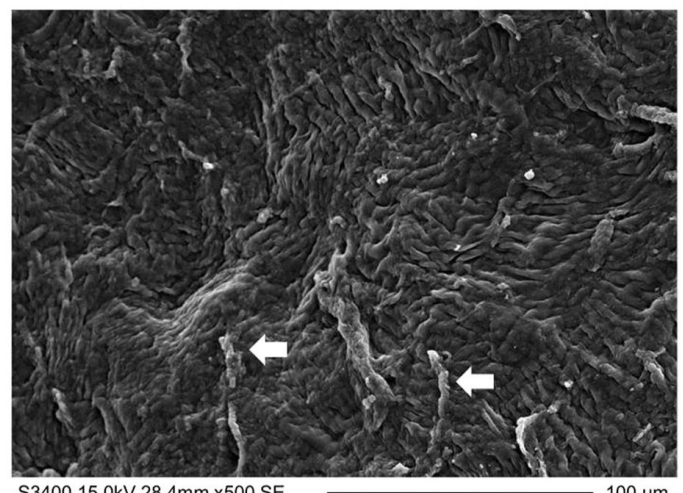

C

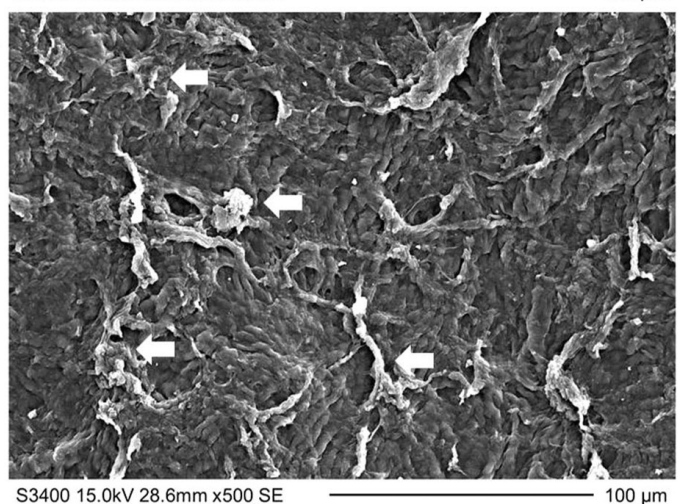

$\mathrm{E}$

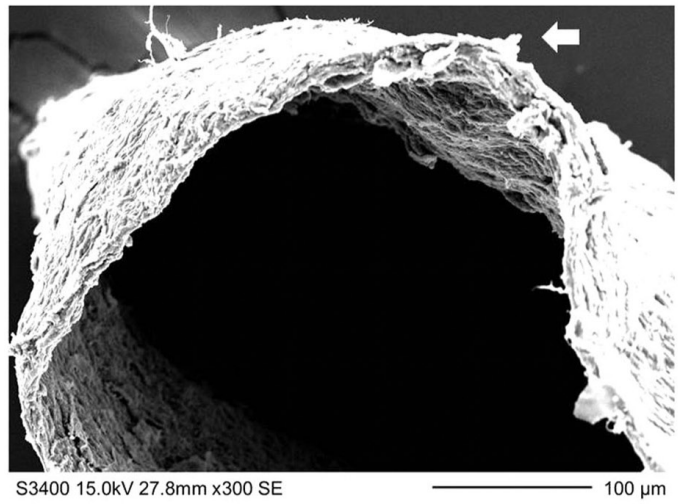

\section{B}

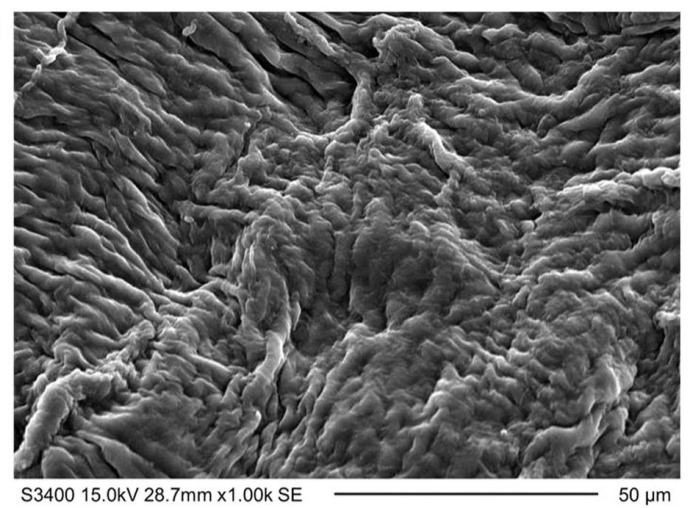

D

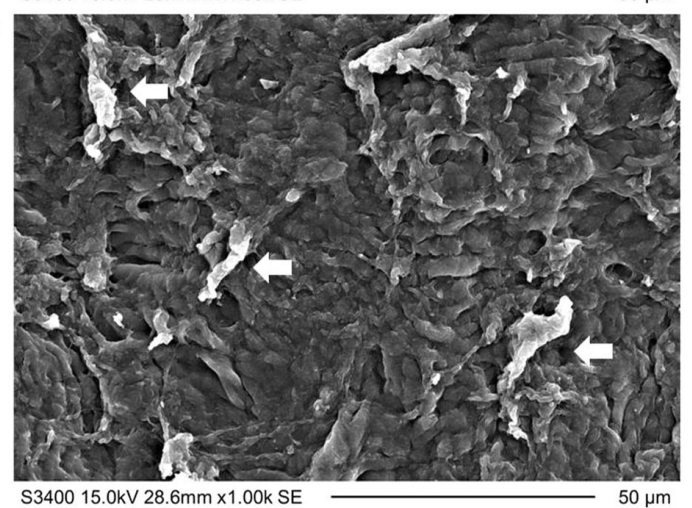

$\mathrm{F}$

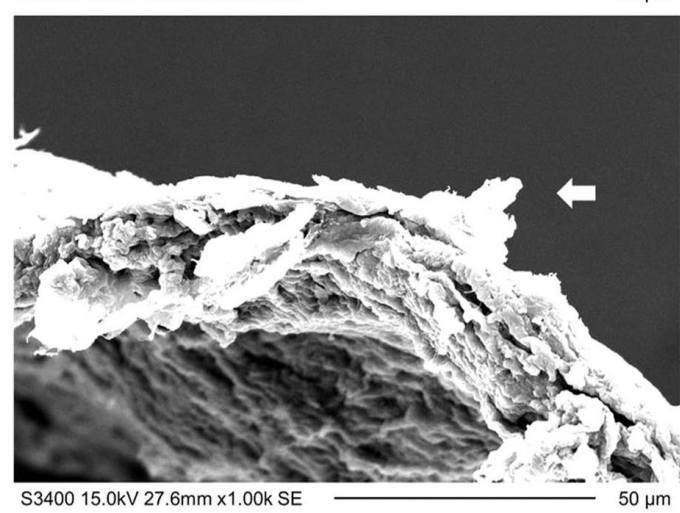

Figure 2. Structure of the front, rear and side edge of the corneal stromal lenticule observed under an electron microscope at different magnifications. Some linear uplifts, short wrinkles and curls, as well as tissue bridges, were observed on the two surfaces of the lenticule (white arrows). (A and B) The front surface was smoother than the $(\mathrm{C}$ and $\mathrm{D})$ rear surface. (E and F) The side edge of the lenticule was rough with short wrinkles, curls and fractured collagens. 

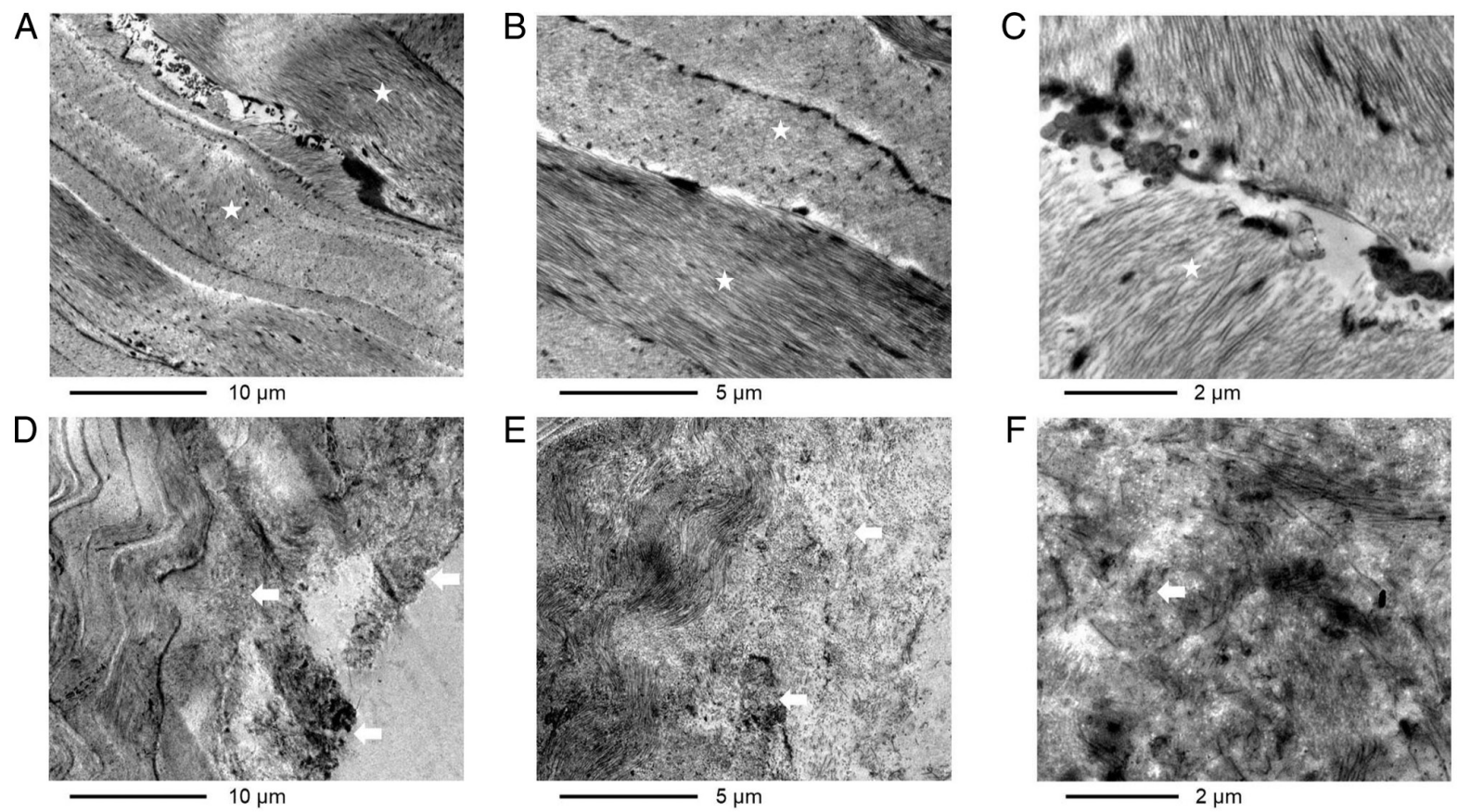

Figure 3. The microscopic observation of the collagen fibers and bundles in the corneal stromal lenticule. (A-C) Under an electron microscope, the collagen fibers in the middle of the lenticule were normally distributed parallel to each other like waves (white asterisks) and the collagen bundles were arranged crosswise. (D-F) The collagen fibers near the surface were disordered with short protuberances, lysis and necrosis (white arrows).
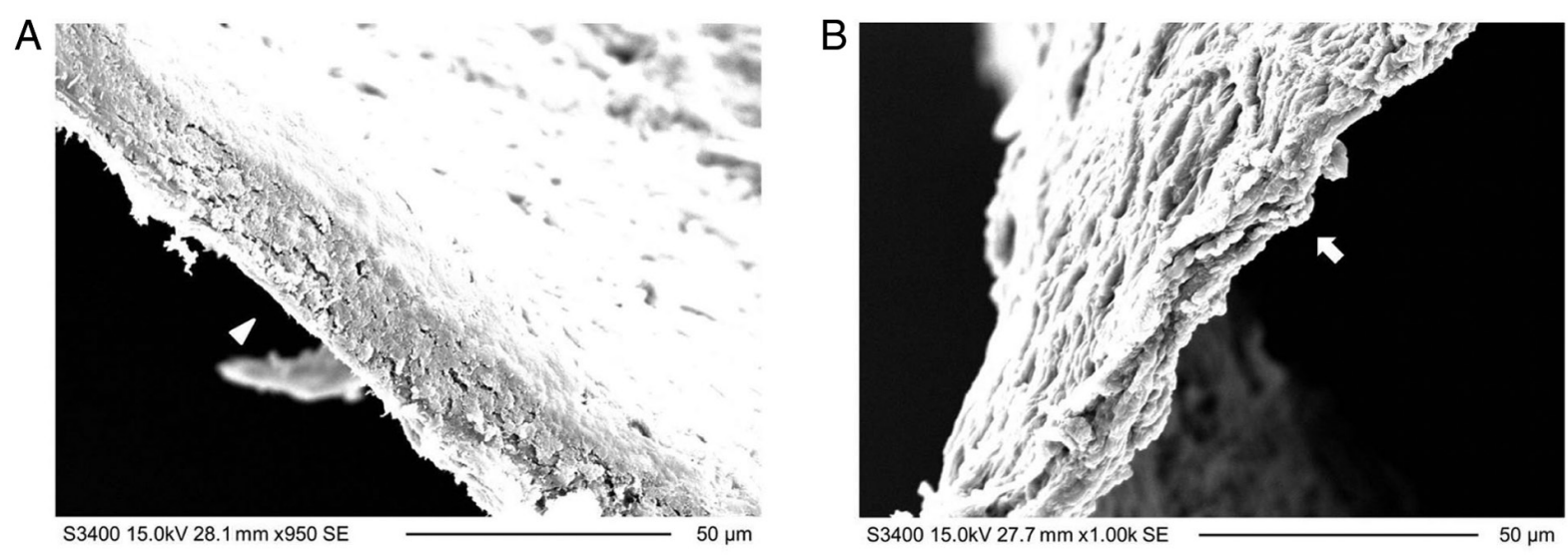

Figure 4. The comparison of the lenticule transection by the microscissor and laser ablation. (A) The transection cut using the microscissor (white arrowhead) was smoother compared with (B) the side edge of the corneal stromal lenticule after laser ablation (white arrow) when observed under an electron microscope.

side edge of the lenticule was rough with short wrinkles, curls and fractured collagen fibers (Fig. 2E and F); iv) in the middle of the lenticule, the corneal stromal fibers were normally distributed in parallel to each other like waves and the collagen bundles were mutually arranged crosswise (Fig. 3A-C); and v) the collagen fibers near the surface were disordered and exhibited short protuberances, lysis and necrosis, and transited to be normal towards the center (Fig. 3D-F).

Quantitative evaluation of the surface quality of the corneal stromal lenticules under the electron microscope. The quality of the front and rear surface of the corneal stromal lenticules was rated based on unified evaluation standards (Table I). As presented in Table II, the smoothness scores of the front surface were markedly higher than those of the rear surface $(\mathrm{P}<0.001)$.
Table II. Quantitative evaluation of the surface quality of corneal stromal lenticules.

\begin{tabular}{lccc}
\hline Score & Front surface & Rear surface & P-value \\
\hline Smoothness & $3.50 \pm 0.33$ & $2.52 \pm 0.24$ & $<0.001^{\mathrm{a}}$
\end{tabular}

${ }^{\text {a }} \mathrm{P}<0.05$ was considered to indicate a statistically significant difference.

Comparison of the side edge cut using a femtosecond laser or microscissors. The corneal stromal lenticule was cut by sharp microscissors and then the transection was compared with the side edge of the lenticule. As presented in Fig. 4, the transection 
cut by the microscissors (Fig. 4B) was smoother than the side edge of the lenticule ablated using a laser (Fig. 4A).

\section{Discussion}

Based on technological improvements, femtosecond lasers have been widely applied in SMILE surgery and have been tentatively used for other corneal surgeries, such as keratoplasty $(12,13)$. In addition, the corneal stromal lenticules extracted during SMILE surgery have been considered in the surgical treatment of hyperopia, corneal ulcers and even corneal perforation, all of which have produced promising clinical results (14-16). It has been reported that the surface quality of the corneal tissue influences wound healing and post-operative visual quality $(8,17,18)$. However, so far, morphological studies regarding the surface quality of human corneal tissue after femtosecond laser scanning are limited. Therefore, it is useful to evaluate the effect of femtosecond lasers on the corneal stromal lenticules by light and electron microscopy, which may help to form a foundation for the future improvement of medical laser technology and corneal surgery using medical lasers.

In the present study, the surface of corneal stromal lenticules was observed to exhibit burrs, broken fibers and tissue bridges under both light and electron microscopes, and was not perfectly smooth at the micro-scale, which was consistent with the results of previous studies $(8,19)$. The underlying mechanisms may be attributed to the effects of femtosecond laser on the corneal stroma. During SMILE surgery, the lenticule surface is the ablating focus of the femtosecond laser and the photo-decomposing function of the femtosecond laser makes it possible to break the chemical bonds between molecules and to produce a number of bubbles. Once the bubbles expand and merge to form a separate plane in the corneal tissue, the corneal stroma may be separated $(20,21)$. However, due to the existence of discontinuous cavitation bubbles, certain tissue bridges remain and the collagen fibers tend to curl and wrinkle, which results in a not-so-smooth surface of the corneal stromal bed. Certain bubbles may be partially absorbed by the surrounding corneal tissue or spread into the air $(22,23)$. The remaining bubbles tend to be fixed in the tissue when preparing the lenticule samples, as was observed in the cross-sections of the lenticules under the light microscope. The observed trends in bubble distribution revealed in the present study may not necessarily be the same as those reported in a previous study (24), in which the bubbles were observed to be preferably located in the anterior layer of the corneal stroma. The latter may be ascribed to the dense collagen fibers in the anterior stroma that markedly limit the movement of bubbles (25). However, in the present study, the lenticule was thin. Thus, the difference in collagen fiber density between the anterior and posterior layers of the lenticules was not significant. Therefore, the preferential bubble distribution could hardly be observed.

In addition to the influence of existing bubbles, other factors, including the laser parameters (26-28), surgical technique (29,30), cap thickness (31-33) and mechanical separation (34), may also affect the surface smoothness. For instance, Kunert et al (26) reported that the surface regularity of corneal lenticules tends to decrease with the increase in laser energy. Serrao et al (27) demonstrated that the stromal interface quality may be improved by decreasing the pulse energy and spot distance. In addition, it has been noticed that a smooth appearance of the lenticular surface exists when a thin cap or a shallow ablating depth is created (31-33). When a laser beam passes through a thick corneal tissue, the focus of the beam is distorted and this weakens the laser efficacy; therefore, additional tissue bridges are created and these make the ablating surface more irregular. Furthermore, it has been speculated that the mechanical separation of the corneal lenticules during SMILE surgery after laser ablation may cause damage to the corneal tissue (34).

The present study further indicated that the front surface of the lenticule was more regular than the rear surface by subjective evaluation according to the unified criteria. This rating method was rapid and economical for evaluating the surface quality and had been widely applied by certain other similar studies $(11,26)$. As to the different regularity between the front and rear surfaces, the primary reason may be that the collagen fibers in the anterior corneal stroma are denser than those in the posterior corneal stroma (25). Therefore, the posterior corneal stroma has less of a restrictive effect on the geometrical shape of bubbles produced by light blasting. Accordingly, the bubbles in the posterior stroma are wider in the vertical direction than those in the anterior stroma; therefore, the bubbles in the posterior stroma are closer to a wide ellipse rather than a long ellipse or even a long shuttle-like shape. Therefore, given the condition of a fixed spot distance, the ability of the bubbles in the posterior stroma to diffuse and fuse with each other along the horizontal direction and form a separation plane was limited, which finally resulted in the formation of tissue bridges, curled fibers and an irregular rear surface of the lenticule. Furthermore, when numerous tissue bridges remain in the posterior stroma after laser scanning, the mechanical separation of the corneal lenticule encounters great resistance, which may cause additional damage to the rear surface of the corneal lenticule.

In addition, the present study revealed that the side edge of the stromal lenticule ablated by a femtosecond laser was not as regular as the transection cut by microscissors, which was similar to the results of a previous study, which compared the cutting surface generated using a femtosecond laser with that generated using a microlamellar knife (7). Due to the damage induced by laser energy, the stromal fibers broke, crinkled and shrunk, making the cutting surface irregular at the micro-level. According to a previous study, no differences in surface roughness were observed between the mechanically resected tissues and those ablated using $0.50 \mu \mathrm{J}$ pulse laser energy (35). However, when the laser pulse energy was $>0.50 \mu \mathrm{J}$, the surface smoothness was no longer predictable and controllable. In addition, the surgical operation of artificially removing the corneal lenticule through a small incision may introduce additional damage to the side edge of the lenticule.

Concerning the wavy change of the stromal fibers and deep-stained margin of the lenticule, as observed under the electron microscope, the biological reactions and thermal injury caused by laser energy may be the primary reasons (36). The high-density free electrons produced by the laser may shrink and expand in the focusing area, which leads to the 
formation of shock waves that generate the wavy change of superficial fibers (37). Furthermore, the electron motions led to an increase in the local temperature and caused thermal damage. Due to thermal injury, the edge was edematous and deep staining was observed under a microscope. The high energy of the laser may also destroy the molecular skeleton and produce bio-molecular fragments, such as free radicals, which tend to destroy or even kill the cells around the laser focusing area (37). Therefore, disordered and necrotic collagen fibers were observed at the edge of the lenticules. Since the thermal radiation range of the femtosecond laser was $<1 \mu \mathrm{m}$, there was no obvious tissue reaction in the deep corneal stroma.

Similar to a previous study (31), the cap thickness was set as $120 \mu \mathrm{m}$ and microscopes were used to observe the lenticule surface quality in the present study. To determine the histological and morphological characteristics of the lenticules, H\&E staining was applied to help observe the changes of collagen fibers and scoring criteria for evaluating the smoothness from the SEM images were applied. Furthermore, the side edge of the lenticule ablated by a femtosecond laser was compared with the side edge cut using the microscissor and certain differences in results were observed. However, there were certain limitations to the present study that should be improved in future studies. First, due to the shrinkage and swelling of the cornea specimens, it was hard to actually effectively evaluate and compare the surface smoothness between different corneal lenticules. It was also difficult to distinguish the causes of lenticule edema between the laser energy and the saving condition of the extracted lenticule, as well as the occurrence of edema prior to or after the femtosecond laser ablation. Furthermore, in the present study, the smoothness of the corneal surfaces was not compared between different laser energies, spot distances, lenticule thicknesses and corneal cap thicknesses. Finally, in the present study, the software applied by Weng et al (31) was not used to evaluate the surface quality of the lenticules, which may have led to the evaluation of the results being affected by the technicians. Additional studies with larger sample sizes are required to obtain a sophisticated comprehension of the most important factors that influence the smoothness of the ablated surface.

In conclusion, it was demonstrated that the surface quality of corneal stromal lenticules ablated using a femtosecond laser was imperfect under a microscope. Additional attention should be focused on achieving an improved laser scanning protocol that may help improve the histology and morphology of the corneal surface following laser ablation. This would be promising, since numerous corneal materials may be used for the treatment of corneal diseases once the corneal stromal lenticules have a perfect surface quality.

\section{Acknowledgements}

Not applicable.

\section{Funding}

This study was supported by the Natural Science Foundation of Hunan Province, China (grant no. 2015JJ4093) and the National Natural Science Foundation of China (grant no. 81900890).

\section{Availability of data and materials}

All data generated or analyzed during this study are included in this published article.

\section{Authors' contributions}

DW, YY and XW (author position 7) designed the study and revised the manuscript. DW also performed the surgery. YY finished the drafting of the manuscript. TH, AX, YF and YZ analyzed and interpreted the data. The histological and microscopic examinations were performed by XW (author position 6). All authors read and approved the final manuscript.

\section{Ethics approval and consent to participate}

This study was approved by the ethics committee of Xiangya Hospital (Changsha, China). Prior to surgery, informed consent was obtained from the myopic patients in accordance with the institutional guidelines after being given a full understanding of the benefits and risks of the surgery as well as the use of their cornea tissues for experimental purpose.

\section{Patient consent for publication}

Not applicable.

\section{Competing interests}

The authors declare that they have no competing interests.

\section{References}

1. Aristeidou A, Taniguchi EV, Tsatsos M, Muller R, McAlinden C, Pineda R and Paschalis EI: The evolution of corneal and refractive surgery with the femtosecond laser. Eye Vis (Lond) 2: 12, 2015.

2. Reinstein DZ, Archer TJ and Gobbe M: Small incision lenticule extraction (SMILE) history, fundamentals of a new refractive surgery technique and clinical outcomes. Eye Vis (Lond) 1: 3 , 2014.

3. Yan H, Gong LY, Huang W and Peng YL: Clinical outcomes of small incision lenticule extraction versus femtosecond laser-assisted LASIK for myopia: A Meta-analysis. Int J Ophthalmol 10: 1436-1445, 2017.

4. Wang D, Liu M, Chen Y, Zhang X, Xu Y, Wang J, To CH and Liu Q: Differences in the corneal biomechanical changes after SMILE and LASIK. J Refract Surg 30: 702-707, 2014.

5. Shah R, Shah S and Sengupta S: Results of small incision lenticule extraction: All-in-one femtosecond laser refractive surgery. J Cataract Refract Surg 37: 127-137, 2011.

6. Chen Y, Yin YW, Zhao Y, Wu XY, Young K, Song WT, Xia XB and Wen D: Differentiation of human embryonic stem cells derived mesenchymal stem cells into corneal epithelial cells after being seeded on decellularized SMILE-derived lenticules. Int J Ophthalmol 12: 717-724, 2019.

7. Soong HK and Malta JB: Femtosecond lasers in ophthalmology. Am J Ophthalmol 147: 189-197.e2, 2009.

8. Sumioka T, Miyamoto T, Takatsuki R, Okada Y, Yamanaka O and Saika S: Histological analysis of a cornea following experimental femtosecond laser ablation. Cornea 33 (Suppl 11): S19-S24, 2014.

9. Miao H, He L, Shen Y, Li M, Yu Y and Zhou X: Optical quality and intraocular scattering after femtosecond laser small incision lenticule extraction. J Refract Surg 30: 296-302, 2014.

10. Heichel J, Blum M, Duncker GI, Sietmann R and Kunert KS: Surface quality of porcine corneal lenticules after Femtosecond Lenticule Extraction. Ophthalmic Res 46: 107-112, 2011. 
11. Cheng YY, Kang SJ, Grossniklaus HE, Pels E, Duimel HJ, Frederik PM, Hendrikse F and Nuijts RM: Histologic evaluation of human posterior lamellar discs for femtosecond laser Descemet's stripping endothelial keratoplasty. Cornea 28: 73-79, 2009.

12. Yoo SH and Hurmeric V: Femtosecond laser-assisted keratoplasty. Am J Ophthalmol 151: 189-191, 2011.

13. Chamberlain WD, Rush SW, Mathers WD, Cabezas M and Fraunfelder FW: Comparison of femtosecond laser-assisted keratoplasty versus conventional penetrating keratoplasty. Ophthalmology 118: 486-491, 2011.

14. Zhao J, Shang J, Niu L, Xu H, Yang D, Zhao Y, Fu D and Zhou X: Two-year outcome of an eye that underwent hyperopic LASIK following inadvertent myopic SMILE lenticule in situ implantation. BMC Ophthalmol 19: 176, 2019.

15. Jin H, He M, Liu H, Zhong X, Wu J, Liu L, Ding H, Zhang C and Zhong X: Small-incision femtosecond laser-assisted intracorneal concave lenticule implantation in patients with keratoconus Cornea 38: 446-453, 2019.

16. Abd Elaziz MS, Zaky AG and El SaebaySarhan AR: Stromal lenticule transplantation for management of corneal perforations; one year results. Graefes Arch Clin Exp Ophthalmol 255: 1179-1184, 2017.

17. Piñero-Llorens DP, Murueta-Goyena Larrañaga A and Hanneken L: Visual outcomes and complications of small-incision lenticule extraction: A review. Exp Rev Ophthalmol 11: 59-75, 2016.

18. Ang M, Chaurasia SS, Angunawela RI, Poh R, Riau A, Tan D and Mehta JS: Femtosecond lenticule extraction (FLEx): Clinical results, interface evaluation, and intraocular pressure variation. Invest Ophthalmol Vis Sci 53: 1414-1421, 2012

19. Zhao J, Miao H, Han T, Shen Y, Zhao Y, Sun L and Zhou X: A Pilot Study of SMILE for Hyperopia: Corneal Morphology and Surface Characteristics of Concave Lenticules in Human Donor Eyes. J Refract Surg 32: 713-716, 2016.

20. Sugar A: Ultrafast (femtosecond) laser refractive surgery. Curr Opin Ophthalmol 13: 246-249, 2002.

21. Vossmerbaeumer U and Jonas JB: Structure of intracorneal femtosecond laser pulse effects in conical incision profiles. Graefes Arch Clin Exp Ophthalmol 246: 1017-1020, 2008.

22. Kaiserman I, Maresky HS, Bahar I and Rootman DS: Incidence, possible risk factors, and potential effects of an opaque bubble layer created by a femtosecond laser. J Cataract Refract Surg 34 417-423, 2008

23. Kanellopoulos AJ and Asimellis G: Digital analysis of flap parameter accuracy and objective assessment of opaque bubble layer in femtosecond laser-assisted LASIK: A novel technique. Clin Ophthalmol 7: 343-351, 2013.

24. Hurmeric V, Yoo SH, Fishler J, Chang VS, Wang J and Culbertson WW: In vivo structural characteristics of the femtosecond LASIK-induced opaque bubble layers with ultrahigh-resolution SD-OCT. Ophthalmic Surg Lasers Imaging 41 (Suppl): S109-S113, 2010.

25. Winkler M, Chai D, Kriling S, Nien CJ, Brown DJ, Jester B, Juhasz T and Jester JV: Nonlinear optical macroscopic assessment of 3-D corneal collagen organization and axial biomechanics. Invest Ophthalmol Vis Sci 52: 8818-8827, 2011.
26. Kunert KS, Blum M, Duncker GI, Sietmann R and Heichel J: Surface quality of human corneal lenticules after femtosecond laser surgery for myopia comparing different laser parameters. Graefes Arch Clin Exp Ophthalmol 249: 1417-1424, 2011.

27. Serrao S, Buratto L, Lombardo G, De Santo MP, Ducoli P and Lombardo M: Optimal parameters to improve the interface quality of the flap bed in femtosecond laser-assisted laser in situ keratomileusis. J Cataract Refract Surg 38: 1453-1459, 2012.

28. Winkler von Mohrenfels C, Khoramnia R, Maier MM, Pfäffl W, Hölzlwimmer G and Lohmann C: Cut quality of a new femtosecond laser system. Klin Monatsbl Augenheilkd 226: 470-474, 2009 (In German).

29. Sarayba MA, Maguen E, Salz J, Rabinowitz Y and Ignacio TS: Femtosecond laser keratome creation of partial thickness donor corneal buttons for lamellar keratoplasty. J Refract Surg 23 $58-65,2007$.

30. Riau AK, Angunawela RI, Chaurasia SS, Tan DT and Mehta JS: Effect of different femtosecond laser-firing patterns on collagen disruption during refractive lenticule extraction. J Cataract Refract Surg 38: 1467-1475, 2012.

31. Weng S, Liu M, Yang X, Liu F, Zhou Y, Lin H and Liu Q: Evaluation of Human Corneal Lenticule Quality After SMILE With Different Cap Thicknesses Using Scanning Electron Microscopy. Cornea 37: 59-65, 2018.

32. Zhang C, Bald M, Tang M, Li Y and Huang D: Interface quality of different corneal lamellar-cut depths for femtosecond laser-assisted lamellar anterior keratoplasty. J Cataract Refract Surg 41: 827-835, 2015.

33. Soong HK, Mian S, Abbasi O and Juhasz T: Femtosecond laser-assisted posterior lamellar keratoplasty: Initial studies of surgical technique in eye bank eyes. Ophthalmology 112: 44-49, 2005.

34. Zhao Y, Li M, Sun L, Zhao J, Chen Y and Zhou X: Lenticule Quality After Continuous Curvilinear Lenticulerrhexis in SMILE Evaluated With Scanning Electron Microscopy. J Refract Surg 31: 732-735, 2015.

35. Lombardo M, De Santo MP, Lombardo G, Schiano Lomoriello D, Desiderio G, Ducoli P, Barberi R and Serrao S: Surface quality of femtosecond dissected posterior human corneal stroma investigated with atomic force microscopy. Cornea 31: 1369-1375, 2012.

36. Ziebarth NM, Lorenzo MA, Chow J, Cabot F, Spooner GJ, Dishler J, Hjortdal JØ and Yoo SH: Surface quality of human corneal lenticules after SMILE assessed using environmental scanning electron microscopy. J Refract Surg 30: 388-393, 2014.

37. Aoki S, Murata H, Matsuura M, Fujino Y, Nakakura S, Nakao Y, Kiuchi Y and Asaoka R: The effect of air pulse-driven whole eye motion on the association between corneal hysteresis and glaucomatous visual field progression. Sci Rep 8: 2969, 2018. 